\title{
Emergence of soliton chirality in a quantum antiferromagnet
}

\author{
HANS-BENJAMIN BRAUN ${ }^{1,2 *}$, JIRI KULDA ${ }^{3}$, BERTRAND ROESSLI ${ }^{4}$, DIRK VISSER ${ }^{5}$, KARL W. KRÄMER $^{6}$, \\ HANS-ULRICH GÜDEL ${ }^{6}$ AND PETER BÖNI ${ }^{7}$
}

${ }^{1}$ UCD School of Physics, University College Dublin, Belfield, Dublin 4, Ireland

${ }^{2}$ Theoretical Physics, ETH-Hönggerberg, CH-8093 Zürich, Switzerland

${ }^{3}$ Institute Laue-Langevin, BP156, 38042 Grenoble Cedex 9, France

${ }^{4}$ Laboratory for Neutron Scattering, ETH Zürich and Paul Scherrer Institute, Villigen PSI, Switzerland

${ }^{5}$ NWO Physics, ISIS Facility, Rutherford Appleton Laboratory, Chilton, Didcot OX11 0QX, UK

${ }^{6}$ Department of Chemistry and Biochemistry, University of Bern, 3000 Bern 9, Switzerland

${ }^{7}$ Department of Physics E21, Technical University Munich, D-85747 Garching, Germany

*e-mail: beni.braun@ucd.ie

L eft- and right-handed chiral matter is present at every scale ranging from seashells to molecules to elementary particles. In magnetism, chirality may be inherited from the asymmetry of the underlying crystal structure, or it may emerge spontaneously. In particular, there has been a long-standing search for chiral spin states ${ }^{1}$ that emerge spontaneously with the disappearance of antiferromagnetic long-range order. Here we identify a generic system supporting such a behaviour and report on experimental evidence for chirality associated with the quantum dynamics of solitons ${ }^{2-5}$ in antiferromagnetic spin chains. The soliton chirality observed by polarized neutron scattering is in agreement with theoretical predictions and is a manifestation of a Berry phase ${ }^{6}$. Our observations provide the first example of the emergence of spin currents and hidden chiral order that accompany the disappearance of antiferromagnetic order, a scheme believed to lie at the heart of the enigmatic normal state of cuprate superconductors.

The disappearance, rather than the appearance, of magnetic order is common to some of the most remarkable magnetic systems. Even when spin chains ${ }^{2,7}$, pyrochlores ${ }^{8}$ and hole-doped antiferromagnets show no apparent long-range magnetic order, the excitations out of the seemingly featureless ground states consist of a rich palette of exotic quasiparticles involving collective behaviour: spin solitons with semion statistics in spin-1/2 chains ${ }^{9}$, spin clusters in pyrochlores ${ }^{10}$ and exotic charge and spin excitations ${ }^{11-14}$ in the normal state of high-transition-temperature (high- $T_{\mathrm{c}}$ ) superconductors. In these systems, the loss of magnetic order is related to the formation of spin singlets, and numerous descriptions build on the influential, albeit controversial, proposal ${ }^{15}$ of using variational wavefunctions based on fluctuating singlets. There has been a long-standing suspicion that fluctuating singlets can give rise to chiral spin correlations ${ }^{1}$, an idea that has, however, remained elusive owing to the complexity of the above systems.

For the present study we investigated a model system that allows us to understand unambiguously the interplay of singlet dynamics, the destruction of antiferromagnetic order and the emergence of chirality. We considered an antiferromagnetic anisotropic spin-1/2 chain, close to the Ising limit. In the classical limit, the ground state is the familiar two-fold degenerate Neel state, with all neighbouring spins aligned antiparallel. The lowest excitation is a domain wall ${ }^{3-5,16}$ separating the two ground states as highlighted by different colours in Fig. 1a. As this domain wall or soliton can be placed anywhere along the chain, the resulting state is highly degenerate. Quantum fluctuations, due to transverse exchange, lift this degeneracy, the soliton becomes delocalized, destroys antiferromagnetic order and forms a band ${ }^{4}$ with two degenerate minima. Remarkably, the states of lowest energy correspond to propagating singlets that carry opposite (vector-) chirality $^{17}$ or spin current,

$$
\mathbf{C}=\sum_{i} \mathbf{S}_{i} \times \mathbf{S}_{i+1}
$$

transverse to the Ising direction. The sum runs over all sites along the chain and $S_{i}$ denotes a spin-1/2 operator at site $i$.

To test such behaviour experimentally, we used spin-polarized neutrons, known for their ability to distinguish right- and left-handed magnetic structures ${ }^{18}$. Here, however, we faced the challenge that spin-1/2 solitons are fractional objects and couple to neutrons only in pairs. As shown in Fig. 1b, a neutron may either create a soliton pair producing a continuum around the energy required to break two bonds, or it scatters an existing thermally excited soliton within the soliton band, producing a low-energy response bounded by the "Villain mode' . Owing to the continuum character of this neutron response, chirality is 

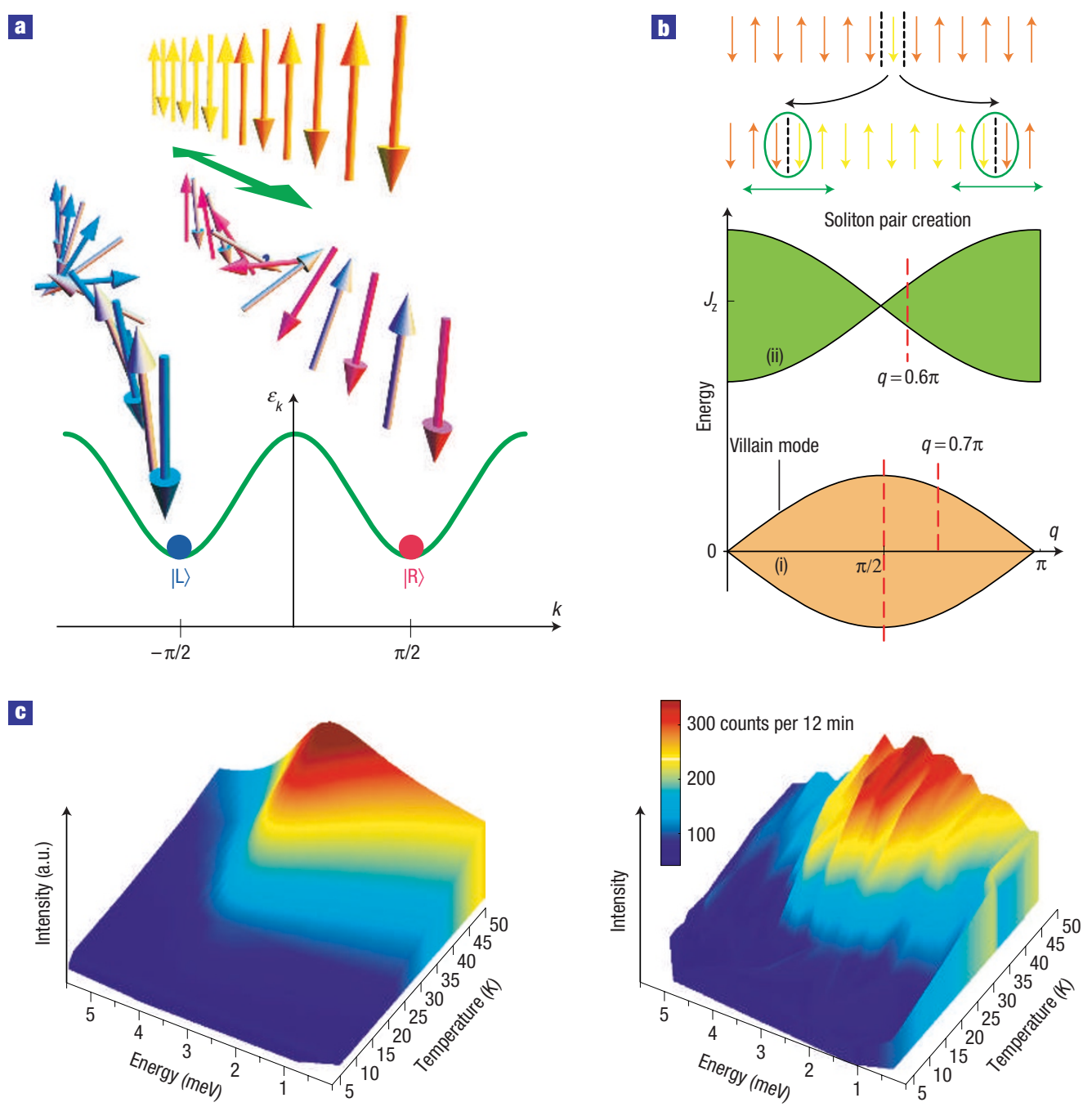

Figure 1 Soliton dispersion, chirality and two-soliton continua contributing to neutron response. a, An Ising soliton is delocalized by quantum fluctuations, leading to a soliton band $\varepsilon_{k}$ with lowest energy states $|\mathrm{L}\rangle,|\mathrm{R}\rangle$ that are left- and right-handed. $\mathbf{b}$, Decay of a magnon into two solitons. A single spin-flip creates the same number of broken bonds as the reversal of a whole spin cluster. Soliton-pair processes give rise to two continua determining the neutron response. (i) Soliton scattering between wavevectors $k_{1}, k_{2}$ in the one-soliton band gives rise to a low-lying continuum, bounded by the 'Villain mode'. This process occurs at fixed neutron wavevector transfer, $q=k_{2}-k_{1}$, for a continuum of energy transfers, $\hbar \omega_{q}=\varepsilon_{k_{2}}-\varepsilon_{k_{1}}$. Dashed lines indicate scans of Figs 2 and 3. (ii) Pair creation of solitons with total wavevector $q=k_{1}+k_{2}$, occurring at energies $\hbar \omega_{q}=\varepsilon_{k_{1}}+\varepsilon_{k_{2}}$. c. Calculated (left) and observed (right) temperature dependence of the low-energy continuum bounded by the Villain mode at $q=0.7 \pi$ for $\mathrm{CsCOBr}_{3}$.

hidden, which explains why it has remained undetected so far. An observation of a chiral signal requires lifting the degeneracy between the contributions of left- and right-handed solitons.

The system we chose is $\mathrm{CsCoBr}_{3}$, the best-known realization of an antiferromagnetic Heisenberg-Ising spin-1/2 chain. Together with its isomorphous twin $\mathrm{CsCoCl}_{3}$, it is well characterized and has been shown to support quantum dynamics of solitons ${ }^{5,19-22}$. In the one-dimensional regime, well above the Néel temperature ${ }^{5}$ $T_{\mathrm{N}}=28.3 \mathrm{~K}$, the interaction between the lowest-lying $\mathrm{Co}^{2+}$ Kramers doublets along a chain is described by the spin-1/2 HeisenbergIsing (XXZ-) hamiltonian,

$$
H=\sum_{i} J_{z} S_{i}^{z} S_{i+1}^{z}+J_{\mathrm{t}}\left(S_{i}^{x} S_{i+1}^{x}+S_{i}^{y} S_{i+1}^{y}\right),
$$

with the exchange constants ${ }^{5} J_{z}=13.8 \mathrm{meV}$ and $J_{\mathrm{t}}=1.9 \mathrm{meV}$. The elementary excitations are solitons rather than conventional magnon excitations of a three-dimensional ordered magnet.
The decay of a magnon into two solitons is illustrated in Fig. $1 b$. Quantum dynamics is induced by the transverse exchange that is proportional to $J_{t}$, which causes adjacent spin pairs to flip. To leading order in $J_{\mathrm{t}} / J_{\mathrm{z}}$, this term simply propagates the soliton by two lattice constants, giving rise to the one-soliton dispersion ${ }^{4,23}$

$$
\varepsilon_{k}=J_{z} / 2+J_{\mathrm{t}} \cos 2 k+g \mu_{\mathrm{B}} B \cos k,
$$

where the first term is the energy of the broken bond and the last term includes the effect of a small magnetic field $\mathbf{B}$ transverse to the Ising direction. Here $k$ is the wavevector, $\mu_{\mathrm{B}}$ is the Bohr magneton and $g$ is the gyromagnetic ratio. The eigenstates of the hamiltonian $H$ are soliton Bloch states $|k\rangle$, consisting of a coherent superposition of solitons at all lattice sites (see Methods). Remarkably, these Bloch states are also eigenstates of the chirality or spin-current operator $^{17}$ satisfying $C_{x}|k\rangle=\sin k|k\rangle$. The states $|\mathrm{R}\rangle,|\mathrm{L}\rangle$ at $k= \pm \pi / 2$ thus have opposite chirality, $\left\langle C_{x}\right\rangle= \pm 1$, as shown 

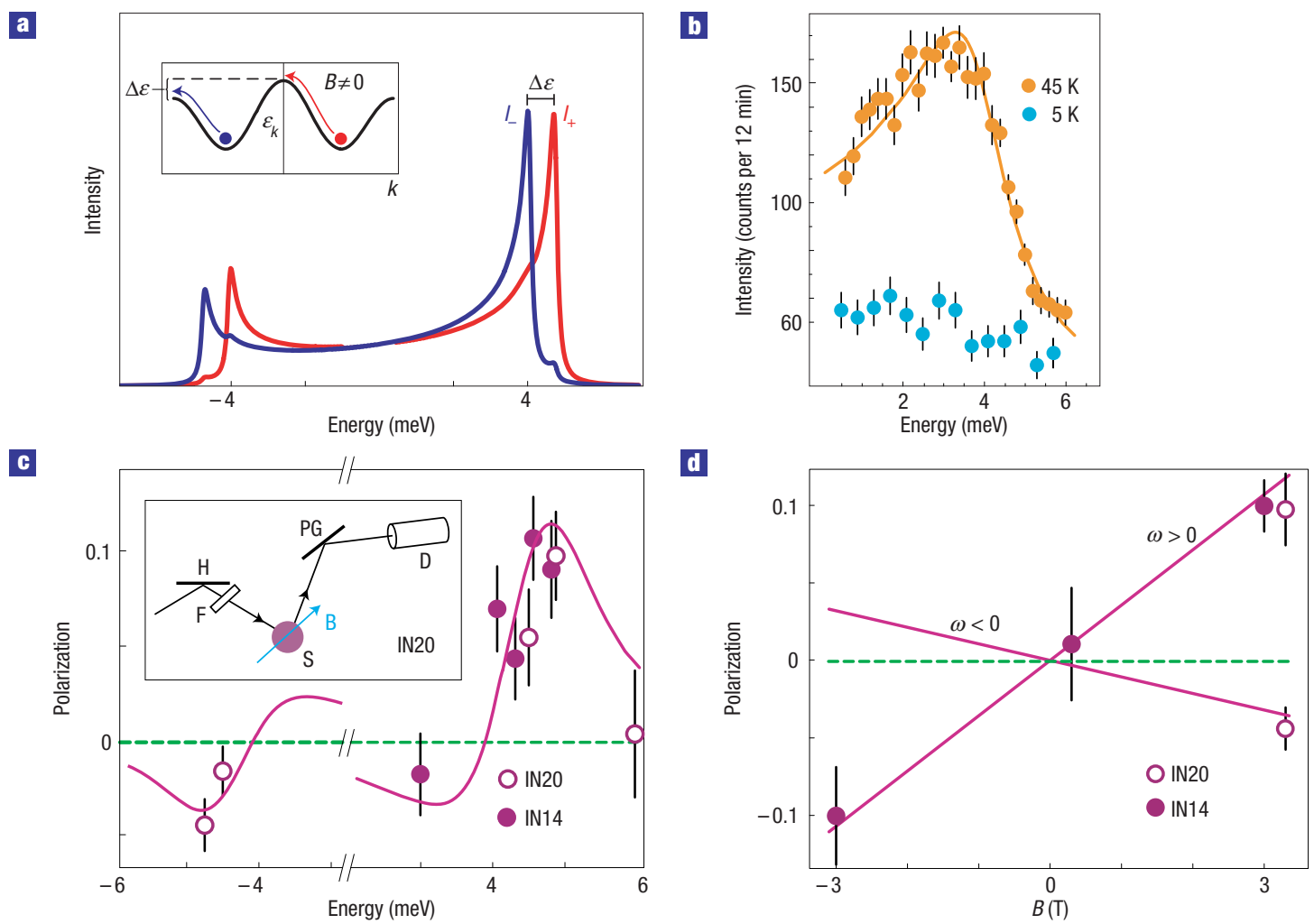

Figure 2 Soliton chirality and the Villain mode in $\mathrm{CsCOBr}_{3}$. a, Theoretical prediction for the Villain-mode splitting due to soliton chirality. $I_{+}\left(I_{-}\right)$denote the scattering intensity for neutrons incident with spin parallel (antiparallel) to the external field. Inset: A weak transverse magnetic field lifts the degeneracy for transitions out of the chiral band minima, $\Delta \varepsilon=2 g \mu_{\mathrm{B}} B$. Arrows indicate transitions of largest spectral weight induced by a neutron momentum transfer $q=\pi / 2$. b. Unpolarized neutron response of the Villain mode at $q=\pi / 2$ in zero field for $T=5$ and $45 \mathrm{~K}$; the solid line is a fit according to ref. 5 . c, Observed polarization $\Pi=\left(I_{+}-I_{-}\right) /\left(I_{+}+I_{-}\right)$indicating soliton chirality in $\mathrm{CsCOBr}_{3}$. Maximal polarization is measured near the energy $\hbar \omega= \pm 2 \mathrm{~J}_{\mathrm{t}}$ of the Villain mode for $q=\pi / 2(\mathrm{Q}=(1.4,0,0.5)$; reciprocal lattice units), $B=3.3 \mathrm{~T}$, $T=45 \mathrm{~K}$. The solid line represents the theoretical result (see Methods). The dashed line indicates zero chirality. Data are taken on spectrometers using cold neutrons (IN14) or thermal neutrons (IN20), respectively. Inset: experimental setup on IN20. The neutron beam is polarized at a Heusler monochromator (H) and passes a spin-flipper (F). No polarization analysis is performed after the beam is scattered on the sample (S). After passing a pyrolytic graphite analyser (PG) the neutrons incident on the detector (D) are counted. d, Integrated polarization near $\pm 4.5 \mathrm{meV}$ as a function of the applied magnetic field. As expected from theory (solid lines, see Methods), polarization reverses with magnetic field $\mathbf{B}$, energy transfer $\hbar \omega$, and vanishes in zero field. Plots are produced directly from raw data without background subtraction. Error bars are statistical, derived from the square root of the number of detector counts (see Methods). The maximal polarization is a $6 \sigma$ effect ( $\sigma$ being the standard deviation) and corresponds to a total counting time on IN14 of approximately $60 \mathrm{~h}$.

in Fig. 1a. Quantum fluctuations due to transverse exchange thus generate soliton chirality from the highly degenerate manifold of static Ising solitons.

Figure 1c shows the excellent agreement between the soliton description and the experimentally measured neutron response in $\mathrm{CsCoBr}_{3}$. At temperatures much lower than $J_{z}$, the soliton band $\varepsilon_{k}$ is unpopulated and the scattering intensity negligible. With increasing temperature, the soliton band becomes thermally populated. Neutrons are scattered off these solitons, thereby inducing inelastic transitions within the soliton band, and the neutron response consists of a continuum, bounded by the intensity maximum at the Villain mode.

Measuring soliton chirality is far more challenging. Whereas experiments traditionally focus on the diagonal part of the susceptibility, here we take advantage of the fact that an incident beam of spin polarized neutrons couples to the antisymmetric part of the susceptibility. Designating $I_{+}\left(I_{-}\right)$as the scattering intensity of neutrons incident with spin parallel (antiparallel) to the applied magnetic field, the signature of soliton chirality becomes a non-vanishing value of the polarization $\Pi \equiv\left(I_{+}-I_{-}\right) /\left(I_{+}+I_{-}\right)$ (see Methods). Note that $I_{+}+I_{-}$is the conventional scattering intensity for unpolarized neutrons, and $\Pi$ would be identically zero for unpolarized incident neutrons or for non-chiral soliton states. As a consequence of the hidden character of chirality, a net polarization becomes observable only when the $\pi$-periodicity of the dispersion $\varepsilon_{k}$ is lifted. This is achieved by the external field $\mathbf{B}$, which alters the dispersion as shown in the inset of Fig. 2a; the main panel of the figure shows the calculated scattering intensities $I_{ \pm}$for $q=\pi / 2$. Even for infinitesimal $B$, soliton chirality gives rise to a finite value of $\Pi$ and hence to a finite real part of the antisymmetric susceptibility ${ }^{18,24}$. The same quantity vanishes at zero field. This property and the identification of chirality and solitons render this effect fundamentally different from studies of systems that show a chiral response either owing to geometric frustration ${ }^{25}$ or owing to a parity breaking (Dzyaloshinski-Moriya) term in the hamiltonian ${ }^{24,26}$. The latter would add a contribution proportional to $\sin k$ to the dispersion $\varepsilon_{k}$ and hence lead to a $\Pi$ of the same sign for positive and negative energy transfers.

Our neutron-scattering experiments on $\mathrm{CsCoBr}_{3}$ were performed at the Institute Laue-Langevin in Grenoble, using the world's most intense beam of polarized neutrons. We first studied the emergence of the Villain mode as a function of temperature 


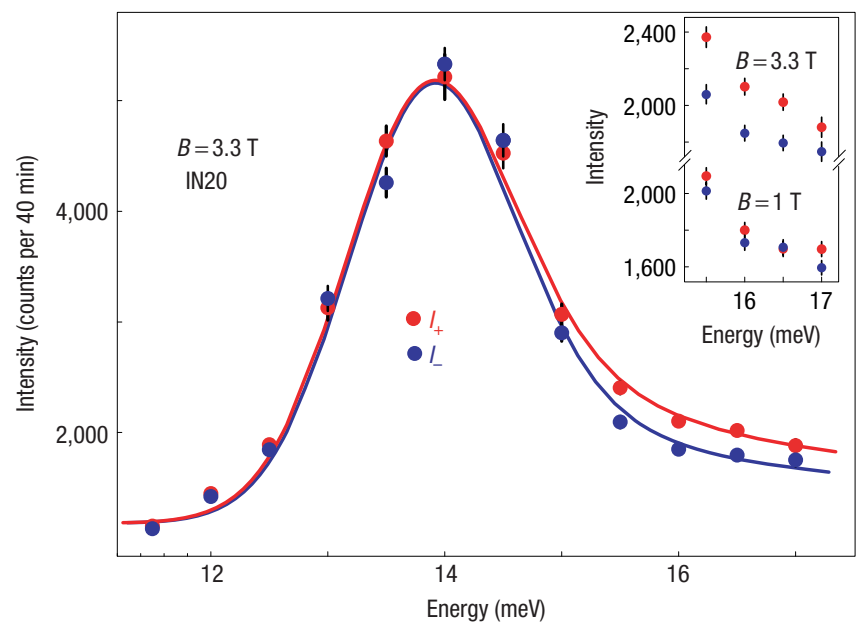

Figure 3 Soliton chirality in the two-soliton continuum. At $q=0.6 \pi$ $(\mathbf{Q}=(2.2,0,1.4)$; reciprocal lattice units), a significant polarization $\Pi$ is observed at high energy around the Ising energy $J_{2}=13.8 \mathrm{meV}$. This high-energy tail corresponds to the excitation of a soliton pair with $k_{1}=0, k_{2} \simeq \pi / 2$, which has definite chirality, see the inset in Fig. 2a. The solid lines are a fit to a step function with power-law decay, suggested by the zero-field results of ref. 16 . The inset shows a detailed view of the field dependence of the polarization. The error bars represent the square root of the number of detector counts.

(see Fig. 1c) and followed the dispersion between $q=\pi / 2$ and the antiferromagnetic point $q=\pi$, in good agreement with existing data $^{5}$. Using a beam of polarized neutrons, incident with spin parallel or antiparallel to a magnetic field $B=3.3 \mathrm{~T}$, which was small compared with the bandwidth $g \mu_{\mathrm{B}} B / 2 J_{\mathrm{t}} \simeq 0.1$, we found the maximal polarization $\Pi$ at $q=\pi / 2$. As shown in Fig. 2 c, the maximal value of $\Pi$ at $q=\pi / 2$ is observed at the energy $4.6 \pm 0.2 \mathrm{meV}$, which is close to the estimated energy $2 J_{\mathrm{t}}+g \mu_{\mathrm{B}} B \simeq 4.4 \mathrm{meV}$ required to excite a soliton from the chiral band minimum to the band maximum (see the inset of Fig. 2a).

This observation of soliton chirality has been tested in several independent ways. First, as shown in Fig. 2c,d, we compared the measured polarization to the theoretical prediction having parameters completely fixed from a fit to the unpolarized data $I_{+}+I_{-}$(see Methods and Supplementary Information, Fig. S1). Second, we examined the behaviour of the polarization as a function of the external field. In zero field, the polarization vanishes; in a reversed field it changes sign, as predicted from equation (2). Third, Fig. 2c,d shows a sign change of the polarization for negative energy transfers. Again, this agrees with the theoretical prediction (see equation (2)) and may be understood from the inset in Fig. 2a. The observation of opposite polarization near $\pm 4.6 \mathrm{meV}$ unambiguously demonstrates the existence of two band minima of opposite chirality.

As a fourth independent test, we investigated whether soliton chirality also affects the neutron response due to soliton-pair creation. Indeed, our observations in Fig. 3a clearly show a polarization at the high-energy tail of the measured neutron intensity. Even though quantitative predictions are not yet available, this asymmetry is intuitively understood using the inset in Fig. 2a. The soliton pair created with $k_{1}=0, k_{2}=\pi / 2$ has a definite chirality and an energy that is higher by $g \mu_{\mathrm{B}} B$ than that of the remaining manifold with the same centre of mass momentum. This completes the evidence for the chiral nature of soliton excitations.

The chiral soliton states can be expressed as singlets moving through a Néel background, $|\mathrm{L}\rangle,|\mathrm{R}\rangle \propto \sum_{m}( \pm i)^{m}|\downarrow \stackrel{\bullet}{\bullet} \downarrow \uparrow \cdots\rangle$,

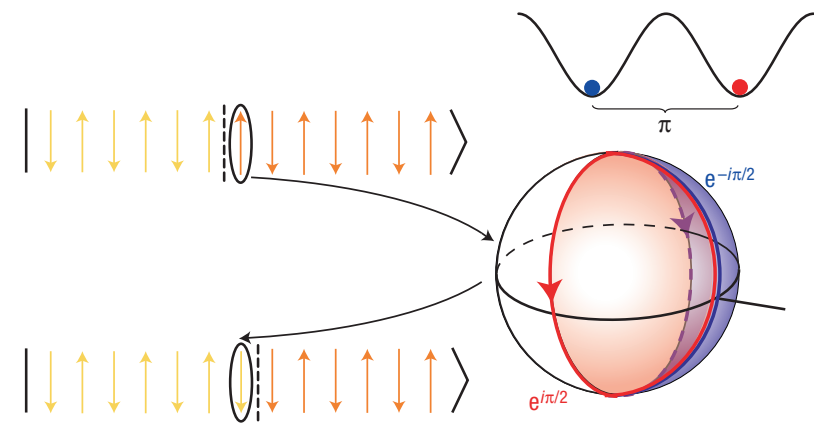

Figure 4 Soliton chirality and Berry's phase. A transition between soliton states centred at nearest-neighbouring sites involves a $\pi$-rotation of one spin. For spin- $1 / 2$, this process involves a Berry phase factor of $\mathrm{e}^{ \pm i \pi / 2}$, depending on whether the spin is rotated clockwise or anticlockwise around the quantization axis. The relative phase between these two states manifests itself as a difference in wavevector of $\pi$ between states of opposite chirality in the soliton dispersion.

where $|\bullet \bullet\rangle=(1 / \sqrt{2})(|\uparrow \downarrow\rangle-|\downarrow \uparrow\rangle)$ is a singlet state of adjacent spins. The resonating character of this state is illustrated by bending an odd-numbered Ising chain into a ring: as the soliton is enforced by boundary conditions, the ground state consists of a singlet that 'resonates' around the ring, reminiscent of the 'resonant valence bond' state proposed for the description of high$T_{\mathrm{c}}$ superconductors ${ }^{15}$. Our observations imply a degeneracy of such states with respect to chirality.

It is illuminating to interpret the observed chirality in terms of Berry's phase ${ }^{6}$. Traditionally, the eigenstates of $H$ are classified according to the total $S_{z}$ component in the Ising direction. Hence they are built from next-nearest-neighbour superpositions of solitons. However, such states can be shown to lead to a vanishing $\Pi$. Our observation of a non-vanishing chirality thus implies that the observed chiral states involve a coherent superposition of solitons at nearest-neighbouring sites, and, remarkably, this remains so even in the limit of an infinitesimal magnetic field. This causes a non-vanishing transition amplitude between soliton states at neighbouring sites, which involves the rotation of one spin-1/2 as shown in Fig. 4. This process must involve a Berry phase factor of $\mathrm{e}^{i \pi / 2}$ or $\mathrm{e}^{-i \pi / 2}$, depending on whether the spin is rotated clockwise or anticlockwise around the quantization $x$ axis. Following the conventional view, one would argue that these phases interfere destructively, causing suppression of nearest-neighbour transitions. However, our observations show that the soliton evades this impending localization at the expense of acquiring a spin-gauge flux $^{27}$, leading to the two chirally distinct excitations $|L\rangle,|R\rangle$ that differ by a wavevector of $\pi$.

This observation has implications that go far beyond the simple system studied here. Berry phases acquired by electrons moving in the effective field of spin textures have been invoked to explain the anomalous Hall effect in pyrochlores ${ }^{28}$. In the context of high- $T_{\mathrm{c}}$ superconductors, the hole motion in a locally antiferromagnetic background involves spin-flips for transitions between adjacent sites $^{29}$, resembling the situation shown in Fig. 4 . Whereas it has been argued that the corresponding Berry phases lead to destructive interference that suppresses nearest-neighbour transitions of holes ${ }^{29}$, our results indicate that this interference is lifted, and a nearest-neighbour transition does occur at the expense of a spin-gauge flux and the emergence of spin currents.

We have observed the spontaneous emergence of chirality and spin currents in the disordered phase of a quantum antiferromagnet, associated with the motion of solitons. This state of matter could be exploited for the generation of spin currents 
in spintronic devices and sheds new light on the elusive nature of quasiparticles in strongly correlated electron systems.

\section{METHODS}

\section{THEORY}

The scattering intensity of a polarized beam of neutrons is given by ${ }^{18}$

$$
I_{ \pm}(\mathbf{Q}, \omega) \propto \int_{-\infty}^{\infty} \mathrm{d} t \mathrm{e}^{-i \omega t}\left\{\left\langle\mathbf{S}_{-\mathbf{Q}}^{\perp} \cdot \mathbf{S}_{\mathbf{Q}}^{\perp}(t)\right\rangle \pm i \hat{\mathbf{x}} \cdot\left\langle\mathbf{S}_{-\mathbf{Q}}^{\perp} \times \mathbf{S}_{\mathbf{Q}}^{\perp}(t)\right\rangle\right\}
$$

where the sign indicates the projection of the spin of the incident neutron onto the $x$ axis parallel to the field direction. Here $\langle\cdots\rangle=\operatorname{tr}\left\{\mathrm{e}^{-\beta H} \cdots\right\} / \operatorname{tr}\left\{\mathrm{e}^{-\beta H}\right\}$ denotes the quantum statistical expectation value. The neutrons transfer momentum $\hbar \mathbf{Q}=\hbar\left(\mathbf{k}_{\mathrm{i}}-\mathbf{k}_{\mathrm{f}}\right)$, where $\mathbf{k}_{\mathrm{i}}$ and $\mathbf{k}_{\mathrm{f}}$ are the wavevectors of the incident and final neutrons respectively, and energy $\hbar \omega$ to the sample. $S_{Q}^{\perp}$ is the Fourier transform of the spin component perpendicular to the momentum transfer. The second chiral term on the right-hand side of equation (1) vanishes in general but is non-zero for helical structures and for chiral soliton states. It is this term that is responsible for the difference between the scattering intensities $I_{+}$and $I_{-}$. This is reflected by a non-zero polarization $\Pi=\left(I_{+}-I_{-}\right) /\left(I_{+}+I_{-}\right)$, with $I_{+}+I_{-}$being the conventional unpolarized scattering intensity. For the Villain mode, the expectation values in equation (1) are evaluated using the exact form of the chiral one-soliton eigenstates $|k\rangle$ with $H|k\rangle=\varepsilon_{k}|k\rangle$. For periodic boundary conditions, $|k\rangle=(1 / \sqrt{2 N}) \sum_{m} \mathrm{e}^{i k m}\left[|m, 1\rangle+\mathrm{e}^{i k N}|m,-1\rangle\right]$, with the sum running over all sites $m$, and $k=\pi v / N, v=-N+1, \ldots, N$, where $N$ is the (odd) number of spins in the chain. Here $|m, 1\rangle=|\downarrow \uparrow \cdot \downarrow \uparrow \underset{m}{\downarrow} \downarrow \uparrow \downarrow \cdot \downarrow \uparrow\rangle$ denotes a soliton at site $m$ of (topological) charge $\rho=1$ and the corresponding antisoliton $|m,-1\rangle$ of charge $\rho=-1$ is obtained by reversing all spins. In the spirit of Villain's approach ${ }^{4}$, we perform the trace in equation (1) within the one-soliton subspace, $\operatorname{tr}\{\cdots\}=\sum_{k}\langle k|\cdots| k\rangle$, and obtain for the chiral part of the cross section,

$$
I_{+}-I_{-}=\frac{I_{0} f}{\cos (q / 2)} \sum_{k} \mathrm{e}^{-\beta \varepsilon_{k}} \sin \left(\frac{k+k^{\prime}}{2}\right) \delta\left(\hbar \omega-\varepsilon_{k}+\varepsilon_{k^{\prime}}\right)
$$

the result for $I_{+}+I_{-}$agrees with the expression that has been previously computed $^{4,5}$ using non-chiral states. Here $k, q$ denote reduced momenta in the chain direction, $\beta=1 / k_{\mathrm{B}} T$ where $k_{\mathrm{B}}$ is the Boltzmann constant and $T$ the temperature, $I_{0}$ is an intensity scale factor that cancels when computing the polarization $\Pi$ and $f$ is a projection factor that depends on the total neutron momentum transfer. The sum in equation (2) extends over all processes where the soliton is scattered from initial wavevector $k$ to $k^{\prime} \equiv k+q-\pi$, and measures the average chirality of the solitons involved in the scattering process. This becomes evident in the limit of $k^{\prime}$ approaching $k$, where each summand measures the chirality of the corresponding soliton state, with maximal contributions at $k= \pm \pi / 2$ and zero contribution at the band maxima $k=0, \pi$. As chirality is hidden, the maximal contributions near $k= \pm \pi / 2$ are of opposite sign and cancel in the absence of external fields. A non-vanishing difference $I_{+}-I_{-}$results only when the $\pi$ periodicity of the dispersion is lifted because of a magnetic field. Fitting the theoretical value of $I_{+}+I_{-}$to the unpolarized data as discussed below, quantitative predictions for $I_{+}, I_{-}$or the polarization can be derived. It is these predictions for $\Pi$ and $I_{ \pm}$that are shown in Fig. 2c,d and in Supplementary Information, Fig. S1, respectively.

\section{EXPERIMENTS}

The neutron-scattering experiments were performed on the three-axis spectrometers IN14 and IN20 at the high-flux reactor of the Institute Laue-Langevin in Grenoble (France). These instruments provide the highest flux of polarized neutrons presently available in their respective energy domains (cold neutrons on IN14 and thermal neutrons on IN20). In short, the wavevector of the incident neutrons, $\mathbf{k}_{\mathrm{i}}$, and their spin polarization are selected by Bragg reflection from a single-crystal Heusler alloy (IN20) or by a pyrolytic graphite (PG 002) monochromator and a supermirror bender (IN14). After passing a spin-flipper, the neutrons arrive at the sample with their spins either parallel or antiparallel to the horizontal magnetic field B. Our high-quality $\mathrm{Cs} \mathrm{CoBr}_{3}$ single crystal with mosaic spread $\eta<20^{\prime}$ and a volume of $2 \mathrm{~cm}^{3}$ was grown using the Bridgman technique. It was placed into a $4 \mathrm{~T}$ horizontal cryomagnet (Oxford Instruments) with the crystallographic axes ( $h 0 l)$ in the scattering plane and $\mathbf{B}$ perpendicular to the crystallographic $c$ axis. To gain intensity, there was no analysis of the polarization and no extra Soller collimators were used on IN14 and IN20. The scattered neutrons were reflected from an analysing Bragg crystal of pyrolytic graphite thus defining the wavevector $\mathbf{k}_{\mathrm{f}}$ at either $k_{\mathrm{f}}=1.5 \AA^{-1}$ (with Be filter) or $2.662 \AA^{-1}$ (PG filter). All data shown in the main figures are produced directly from raw data without performing any background subtraction. All error bars are statistical in nature and derived from the square root of the detector counts. The unpolarized data are fitted with $I_{+}+I_{-}$as discussed above, convoluted with a lorentzian ${ }^{20}$ to account for finite-lifetime effects and convoluted with the instrument resolution function, using Institute Laue-Langevin in-house software. The fitting parameters are background, peak position, intensity and peak width. These parameters based on the unpolarized data are then used to predict the intensities $I_{+}\left(I_{-}\right)$as shown by red (blue) in Supplementary Information, Fig. S1, or $\Pi$ shown in Fig. 2c. These predicted intensities contain no free parameters.

Received 1 July 2005; accepted 30 September 2005; published 6 November 2005.

References

1. Wen, X. G., Wilczek, F. \& Zee, A. Chiral spin states and superconductivity. Phys. Rev. B 39, 11413-11423 (1989).

2. Takahashi, M. Thermodynamics of One Dimensional Solvable Models (Cambridge Univ. Press, Cambridge, 1999)

3. Brooke, J., Rosenbaum, T. F. \& Aeppli, G. Tunable quantum tunneling of magnetic domain walls. Nature 413, 610-613 (2001).

4. Villain, J. Propagative spin relaxation in the Ising-like antiferromagnetic linear chain. Physica B 79, 1-12 (1975).

5. Nagler, S. E., Buyers, W. J. L., Armstrong, R. L. \& Briat, B. Propagating domain walls in $\mathrm{CsCoBr}_{3}$. Phys. Rev. Lett. 49, 590-592 (1982).

6. Berry, M. V. Quantal phase factors accompanying adiabatic changes. Proc. R. Soc. Lond. A 392, 45-57 (1984).

7. Affleck, I. Quantum spin chains and the Haldane gap. J. Phys. C 1, 3047-3072 (1989).

8. Bramwell, S. T. \& Gingras, M. J. P. Spin ice states in frustrated magnetic pyrochlore materials. Science 294, 1495-1501 (2002).

9. Haldane, F. D. M. "Fractional statistics" in arbitrary dimensions: A generalization of the Pauli principle. Phys. Rev. Lett. 67, 937-940 (1991).

10. Lee, S. - H. et al. Emergent excitations in a geometrically frustrated magnet. Nature 418, 856-858 (2002).

11. Christensen, N. B. et al. Dispersive excitations in the high-temperature superconductor $\mathrm{La}_{2-x} \mathrm{Sr}_{x} \mathrm{CuO}_{4}$. Phys. Rev. Lett. 93, 147002 (2004).

12. Hayden, S. M., Mook, H. A., Dai, P. C., Perring, T. G. \& Dogan, F. The structure of the high-energy spin excitations in a high-transition-temperature superconductor. Nature 429, 531-534 (2004).

13. Hinkov, V. et al. Two-dimensional geometry of spin excitations in the high-transition-temperature superconductor $\mathrm{YBa}_{2} \mathrm{Cu}_{3} \mathrm{O}_{6+x}$. Nature 430, 650-653 (2004).

14. Tranquada, J. M. et al. Quantum magnetic excitations from stripes in copper oxide superconductors. Nature 429, 534-538 (2004).

15. Anderson, $\mathrm{P}$. W. The resonating valence bond in $\mathrm{La}_{2} \mathrm{CuO}_{4}$ and superconductivity. Science 235, 1196-1198 (1987).

16. Bougourzi, A. H., Karbach, M. \& Müller, G. Exact two-spinon dynamic structure factor of the one-dimensional $s=\frac{1}{2}$ Heisenberg-Ising antiferromagnet. Phys. Rev. B 57, 11429-11438 (1998).

17. Braun, H. B. \& Loss, D. Chirality correlations of spin solitons: Bloch walls, spin- $\frac{1}{2}$ solitons and holes in a 2D antiferromagnetic background. Int. J. Mod. Phys. B 10, 219-234 (1996).

18. Maleyev, S. V. Investigation of spin chirality by polarized neutrons. Phys. Rev. Lett. 75, 4682-4685 (1996).

19. Nagler, S. E., Buyers, W. J. L., Armstrong, R. L. \& Briat, B. Ising-like spin- $\frac{1}{2}$ quasi-one-dimensional antiferromagnets: Spin-wave response in $\mathrm{CsCoX}_{3}$ salts. Phys. Rev. B 27, 1784-1799 (1983).

20. Boucher, J. P. et al. Solitons in the paramagnetic and partially disordered phases of $\mathrm{CsCoCl}_{3}$. Phys. Rev. B 31, 3015-3026 (1985).

21. Goff, J. P., Tennant, D. A. \& Nagler, S. E. Exchange mixing and soliton dynamics in the quantum spin chain $\mathrm{CsCoCl}_{3}$. Phys. Rev. B 52, 15992-16000 (1995).

22. Tun, Z., Gaulin, B. D., Rogge, R. B. \& Briat, B. Critical soliton dynamics in $\mathrm{CsCoBr}_{3}$. J. Magn. Magn Mater. 104-107, 1045-1046 (1992)

23. Devreux, F. \& Boucher, J. P. Solitons in Ising-like quantum spin chains in a magnetic field: a second quantization approach. J. Phys. 48, 1663-1670 (1987).

24. Roessli, B., Böni, P., Fischer, W. E. \& Endoh, Y. Chiral fluctuations in MnSi above the Curie temperature. Phys. Rev. Lett. 88, 237204 (2002).

25. Plakhty, V. P., Kulda, J., Visser, D., Moskvin, E. V. \& Wosnitza, J. Chiral critical exponents of the triangular-lattice antiferromagnet $\mathrm{CsMnBr}_{3}$ as determined by polarized neutron scattering. Phys. Rev. Lett. 85, 3942-3945 (2000)

26. Coldea, R. et al. Direct measurement of the spin Hamiltonian and observation of condensation of magnons in the 2D frustrated quantum magnet $\mathrm{Cs}_{2} \mathrm{CuCl}_{4}$. Phys. Rev. Lett. 88, 137203 (2002).

27. Braun, H. B. \& Loss, D. Berry's phase and quantum dynamics of ferromagnetic solitons. Phys. Rev. B 53, 3237-3255 (1996)

28. Taguchi, Y., Oohara, Y., Yoshizawa, H., Nagaosa, N. \& Tokura, Y. Spin chirality, Berry phase and anomalous Hall effect in a frustrated ferromagnet. Science 291, 2573-2576 (2001).

29. Auerbach, A. Interacting Electrons and Quantum Magnetism (Springer, Berlin, 1994).

30. Wolf, S. A. et al. Spintronics: A spin-based electronics vision for the future. Science 294 1488-1495 (2001).

\section{Acknowledgements}

We acknowledge illuminating discussions with C. Broholm, J. M. D. Coey, M. Enderle, C. Helm D. Loss, G. Müller, T. M. Rice and M. Sigrist. We thank the Institute Laue-Langevin technical staff, in particular A. Brochier, P. Flores and J. L. Ragazzoni, for their excellent support. This work was supported by the Swiss National Science Foundation, the Center for Theoretical Studies (ETHZ), Enterprise Ireland (IC/2005/0043) and the Science Foundation of Ireland under the Research Frontiers Programme (05/RFP/PHY0023).

Correspondence and requests for materials should be addressed to H.-B.B.

Supplementary Information accompanies this paper on www.nature.com/naturephysics.

\section{Competing financial interests}

The authors declare that they have no competing financial interests.

Reprints and permission information is available online at http://npg.nature.com/reprintsandpermissions/ 